\title{
Vertex Coverings by Monochromatic Paths and Cycles
}

A. Gyárfás

COMPUTER AND AUTOMATION INSTITUTE, HUNGARIAN ACADEMY OF SCIENCES BUDAPEST, HUNGARY

\section{ABSTRACT}

We survey some results on covering the vertices of 2-colored complete graphs by two paths or by two cycles of different color. We show the role of these results in determining path Ramsey numbers and in algorithms for finding long monochromatic paths or cycles in 2-colored complete graphs.

A graph is 2-colored if each edge is colored either red or blue. We consider results on covering the vertices of 2-colored complete (undirected, directed, or bipartite) graphs by monochromatic paths or cycles. Algorithmic proofs of these results are given, and these results are applied to the calculation of generalized Ramsey numbers.

A 2-colored path or cycle is called simple if it is monochromatic or it is the union of a red and a blue path. The following simple result was mentioned in a footnote in [2].

Theorem 1. A 2-colored complete graph $K_{n}$ contains a simple Hamiltonian path.

Proof (Algorithm 1). Assume that $n \geqq 2$. We define simple paths $P_{i}$, $1 \leqq i \leqq n$, in a 2 -colored complete graph $K_{n}^{i}$. Let $P_{1}=x_{1}$ and $P_{2}=x_{1}, x_{2}$, where $x_{1}$ and $x_{2}$ are arbitrary vertices of $K_{n}$. If $2 \leqq i<n$ and $P_{i}$ is already defined, we choose an arbitrary vertex $x_{i+1}$ from $K_{n}-P_{i}$. The symbol $c(x, y)$ denotes the color of the edge $x y$. The path $P_{i+1}$ is defined as follows:

If $c\left(x_{1}, x_{2}\right)=c\left(x_{i-1}, x_{i}\right)\left(P_{i}\right.$ is monochromatic) or $c\left(x_{i-1}, x_{i}\right)=c\left(x_{i}, x_{i+1}\right)$ then $P_{i+1}=x_{1}, x_{2}, \ldots, x_{i}, x_{i+1}$. Stop. If $c\left(x_{i+1}, x_{1}\right)=c\left(x_{1}, x_{2}\right)$ then $P_{i+1}=$ 
$x_{i+1}, x_{1}, \ldots, x_{i}$. Stop. If $c\left(x_{i}, x_{1}\right)=c\left(x_{1}, x_{i+1}\right)$ then $P_{i+1}=x_{2}, \ldots, x_{i}, x_{1}$,

$x_{i+1}$. Stop. If $c\left(x_{i+1}, x_{i}\right)=c\left(x_{i}, x_{1}\right)$ then $P_{i+1}=x_{i+1}, x_{i}, x_{1}, \ldots, x_{i-1}$.

Stop.

It is easy to check that $P_{i+1}$ is simple; therefore $P_{n}$ is a simple Hamiltonian path.

Corollary 1 ([5]). A 2-colored complete graph $K_{n}$ contains a simple Hamiltonian cycle if $n \geqq 3$.

A "vertex covering theorem," like Theorem 1 or Corollary 1 , clearly provides a means to say something about the corresponding Ramsey numbers. Corollary 1 implies that a 2-colored $K_{m+n-3}$ contains a red $P_{m}$ or a blue $P_{n}$ if $3 \leqq m \leqq n$, i.e., $R\left(P_{m}, P_{n}\right) \leqq m+n-3$. This upper bound comes very easily compared to the proof of its exact value, $n+\lfloor m / 2\rfloor-1$ ([2]). Another advantage is that Algorithm 1 is extremely simple and fast for finding a simple Hamiltonian path in a 2-colored $K_{n}$. It is easy to see that the number of elementary steps (comparisons, bookkeeping) to define $P_{i+1}$ in Algorithm 1 does not depend on $i$. This observation gives the following result.

Proposition 1. Algorithm 1 finds a simple Hamiltonian path in a 2-colored $K_{n}$ in $O(n)$ time. Consequently, it finds a monochromatic path of length at least $n / 2$ in $O(n)$ time.

The next result deals with cycles covering a 2 -colored $K_{n}$. It is convenient for our purpose to consider vertices and edges as cycles, a vertex is considered either as a red or a blue cycle, and an edge is considered as a cycle in its color.

Theorem 2. The vertices of a 2-colored $K_{n}$ can be covered by the vertices of one red and one blue cycle, such that the two cycles have at most one common vertex.

Proof (Algorithm 2). The initial step is to construct a simple Hamiltonian cycle $C$ in $K_{n}$ using Algorithm 1 (we assume $n \geqq 3$ ). If $C$ is monochromatic then we stop: $C$ and any vertex of $C$ give the required cycles. We can assume that $C$ is the union of a red and a blue path: $C=R \cup B, R=x_{1}, x_{n}$, $x_{n-1}, \ldots, x_{m}$ and $B=x_{1}, x_{2}, \ldots, x_{m}$ for some $1<m \leqq n$. Since the role of the edge $e=x_{1} x_{m}$ is symmetric in $C$, we may assume that $e$ is red.

Starting from the red cycle $C_{0}=R \cup\{e\}$, we construct red cycles $C_{i}$ for $i=1,2, \ldots$ on the vertex set $x_{m-i}, x_{m-i+1}, \ldots, x_{n}, x_{1}, \ldots, x_{i}, x_{i+1}$, such that $x_{i+1} x_{m-i}$ is an edge of $C_{i}$. If $C_{i}$ is already defined for some $i$ and $\left|C_{i}\right| \geqq n-2$ then we stop: $C_{i}$ and $K_{n}-C_{i}$ (a vertex or a blue edge) give the required cycles. If $\left|C_{i}\right| \leqq n-3$ then we stop when at least one of the following three conditions occurs: 

(A) $x_{i+1} x_{m-i-1}$ is blue: $C_{i}$ and $x_{i+1}, \ldots, x_{m-i-1}$ give the cycles.
(B) $x_{i+2} x_{m-i}$ is blue: $C_{i}$ and $x_{i+2}, \ldots, x_{m-i}$ give the cycles.
(C) $x_{i+2} x_{m-i-1}$ is blue: $C_{i}$ and $x_{i+2}, \ldots, x_{m-i-1}$ give the cycles.

The red and blue cycles are disjoint in case $C$; they have one common vertex in cases $A$ and $B$.

If the conditions $A, B$, and $C$ all fail then $C_{i+1}$ is defined by removing the edge $x_{i+1} x_{m-i}$ from $C_{i}$ and adding the red edges $x_{i+1} x_{m-i-1}, x_{i+2} x_{m-i}$ and $x_{i+2} x_{m-i-1}$. The construction eventually stops since $\left|C_{i+1}\right|=\left|C_{i}\right|+2$.

It is not known whether a 2 -colored $K_{n}$ has a vertex cover by two disjoint (red and blue) cycles. We conjecture that the answer is yes and investigations of special cases in [1] seem to support this.

The analysis of Algorithm 2 gives the following.

Proposition 2. Algorithm 2 finds in $O(n)$ time a vertex cover of a 2-colored $K_{n}$ by two (red and blue) cycles, having at most one common vertex. As a consequence, Algorithm 2 finds in $O(n)$ time a monochromatic cycle of length at least $n / 2$ in a 2 -colored $K_{n}$.

Propositions 1 and 2 show that the problem of finding a monochromatic path or cycle of length at least $n / 2$ in a 2-colored $K_{n}$ is of $O(n)$ complexity, a somewhat surprising fact since the problem input, a 2-colored $K_{n}$, contains $\left(\begin{array}{l}n \\ 2\end{array}\right)$ bits of information. On the other hand, the algorithm coming from the proof of the path-path Ramsey number ([2]) finds in $O\left(n^{2}\right)$ time a monochromatic path of length at least $2 n / 3$ in a 2 -colored $K_{n}$. These considerations lead us to a question.

Problem 1. Is there a real number $c>1 / 2$ and an algorithm $\mathrm{A}$ of $O(n)$ time complexity, such that A finds a monochromatic path of length at least $c n$ in any 2-colored $K_{n}$ ?

The next result (conjectured by Lehel and proved by Raynaud in [5]) generalizes Corollary 1 for complete symmetric directed graphs. (A directed graph $G$ is symmetric if $x y \in E(G)$ implies $y x \in E(G)$.)

Theorem 3. A 2-colored complete symmetric directed graph with at least two vertices contains a simple directed Hamiltonian cycle.

Proof (Raynaud's proof with simplifications). Let $k$ and $m$ be natural numbers satisfying $1 \leqq k \leqq m$, where $k \neq m-1$. A loop $L=L\left(x_{1}, x_{2}, \ldots\right.$, $\left.x_{m} ; k\right)$ is a 2-colored symmetric directed graph on vertices $x_{1}, x_{2}, \ldots, x_{m}$, with red edges $x_{1} x_{2}, x_{2} x_{3}, \ldots, x_{m-1} x_{m}$ and $x_{m} x_{k}$ if $m \neq k$. The "reverse" edges $x_{2} x_{1}, x_{3} x_{2}, \ldots, x_{m} x_{m-1}$ and $x_{k} x_{m}$ (if $m \neq k$ ) are blue. Note the special loops: the one vertex graph $(k=m=1)$, the path $(k=m)$ and the cycle $(k=1$, 
$m \geqq 3$ ). The loops of a 2-colored complete symmetric directed graph have a natural partial ordering:

$$
L_{1} \leqq L_{2} \text { if } L_{1}=L_{1}\left(x_{1}, \ldots, x_{m} ; k_{1}\right), L_{2}=L_{2}\left(x_{1}, \ldots, x_{n} ; k_{2}\right) \text { and } m<n \text { or }
$$
$m=n$ and $k_{2} \leqq k_{1}$.

The proof proceeds by induction on the number of vertices of the 2-colored complete symmetric directed graph $G$. The case $|V(G)|=2$ is trivial. For $|V(G)|>2$, let $L=L\left(x_{1}, \ldots, x_{m} ; k\right)$ be a maximal loop in the partial order defined above and let $H=G-\left\{x_{k}, \ldots, x_{m}\right\}$. If $H$ is empty, then $k=1$ and the red (or blue) edges of $L$ form a Hamiltonian cycle of $G$. If $H$ is nonempty, we apply the inductive hypothesis: let $C=\left\{y_{1}, y_{2}, \ldots, y_{n}\right\}$ be a simple Hamiltonian cycle of $H$. We may assume that $y_{n}$ is the end-vertex of the red path of $C$ (in a monochromatic $C, y_{n}$ can be any vertex).

We observe that either $y_{n} x_{m}$ is a red edge or $x_{m} y_{n}$ is a blue edge in $G$ by the maximality of $L$. If $y_{n} x_{m}$ is red, then $y_{n} x_{m} x_{k} \ldots x_{m-1} y_{1} \ldots y_{n}$ is a simple Hamiltonian cycle of $G$. If $x_{m} y_{n}$ is blue, then $y_{n} y_{1} \ldots y_{n-1} x_{m-1} \ldots x_{k} x_{m} y_{n}$ is a simple Hamiltonian cycle of $G$.

Theorem 3 gives the value of a Ramsey number, $R\left(\vec{P}_{m}, \vec{P}_{n}\right)$, which is the smallest $t$ such that a 2-colored complete symmetric directed graph $\vec{K}_{t}$ always contains either a red $\vec{P}_{m}$ or a blue $\vec{P}_{n}$. An obvious example shows that $. R\left(\vec{P}_{m}, \vec{P}_{n}\right) \geqq m+n-3$ for $m, n \geqq 3$. On the other hand Theorem 3 implies that $R\left(\vec{P}_{m}, \vec{P}_{n}\right) \leqq m+n-3$ for $m, n \geqq 3$.

Corollary 2 ([4], [6]). $R\left(\vec{P}_{m}, \vec{P}_{n}\right)=m+n-3$. for $m, n \geqq 3$.

It is easy to formulate the proof of Theorem 3 as an algorithm to find a simple Hamiltonian cycle in a 2 -colored $\vec{K}_{n}$. Although the algorithm we obtain is still simple (compared to Algorithm 1 for the undirected case), its time complexity is $O\left(n^{2}\right)$. The reason for this behavior is that one needs $O\left(n^{2}\right)$ time to find a maximal loop in a 2-colored $\vec{K}_{n}$.

Path-path Ramsey numbers for complete bipartite graphs were independently established in [3] and [4]. The heart of the method in [4] can be stated as a vertex covering result (Theorem 4 below). An exceptional coloring of a complete bipartite graph with vertex classes $\mathrm{A}$ and $\mathrm{B}$ is a coloring, where $A=A_{1} \cup A_{2}, \quad B=B_{1} \cup B_{2}, A_{1} \cap A_{2}=\emptyset, \quad B_{1} \cap B_{2}=\emptyset$, moreover the edges between $A_{1}$ and $B_{1}, A_{2}$ and $B_{2}$ are all red, the edges between $A_{1}$ and $B_{2}$, $A_{2}$ and $B_{1}$ are all blue.

Theorem 4. A 2-colored complete bipartite graph $K(n, n)$ has either an exceptional coloring or contains a simple path $P$ with the following properties: both the red and the blue path of $P$ have an even number of vertices, $P$ covers the vertices of $K(n, n)$ with one possible exception.

Theorem 4 is suitable to obtain the path-path Ramsey numbers for 2colored complete bipartite graphs. Its simplest consequence is the following corollary. 
Corollary 3. A 2-colored $K(m+n-1, m+n-1)$ contains a red $P_{2 m}$ or a blue $P_{2 n}$.

The proof of Theorem 4 in [4] can be formulated as an $O\left(n^{2}\right)$ time algorithm to find the required simple path or the exceptional covering of a 2-colored $K(n, n)$. This algorithm is not presented here since it is longer and less illustrative than the algorithms shown in this paper.

We have seen that an $O(n)$ algorithm can find a "long" monochromatic path in a 2-colored $K_{n}$ but only $O\left(n^{2}\right)$ algorithms are available for the same purpose if $K_{n}$ is replaced by $\vec{K}_{n}$ or by $K(n, n)$. It would be interesting to know whether an $O(n)$ algorithm can have any power on $\vec{K}_{n}$ and $K(n, n)$. More precisely, we have the following problem.

Problem 2. Is there a positive real number $c$ and an algorithm $A_{1}\left(A_{2}\right)$ of $O(n)$ time complexity such that $A_{1}\left(A_{2}\right)$ finds a monochromatic path of length at least cn in any 2 -colored $\vec{K}_{n}(K(n, n))$ ?

\section{References}

[1] J. Ayel, Sur l'existence de deux cycles supplementaires unicolorés, disjoints et de couleurs differentes dans un graphe complet bicolore. Thesis, University of Grenoble (1979).

[2] L. Gerencsér and A. Gyárfás, On Ramsey-type problems. Ann. Univ. Sci. Bud. de Rol. Eötvös Sect. Math. 10(1967) 167-170.

[3] R. J. Faudree and R. H. Schelp, Path-path Ramsey numbers for the complete bipartite graph. J. Combinatorial Theory Ser. B (1975) 161-173.

[4] A Gyárfás and J. Lehel, A Ramsey-type problem in directed and bipartite graphs. Periodica Math. Hung. 3(1973) 299-304.

[5] H. Raynaud, Sur le circuit hamiltonien bi-colore dans les graphes orientés. Periodica Math. Hung. 3(1973) 289-297.

[6] J. E. Williamson, A Ramsey-type problem for paths in digraphs. Math. Ann. 203(1973) 117-118. 